\title{
Screening for otologic functional impairments in the elderly: whose job is it anyway?
}

B.E. Weinstein

Graduate Center, CUNY, New York, NY, USA

\begin{abstract}
Among the most prevalent of chronic conditions affecting older adults globally, hearing loss prevalence is increasing and its impact on society growing. Untreated hearing loss diminishes ones ability to communicate and its strong association with depression and cognitive decline adds further to the burden of hearing loss. Hearing health care is rarely included in the traditional medical exam for older adults, it is typically not considered a risk factor for cognitive decline or falls, and it is not a condition for which routine screening has been recommended by the U.S. Preventive Services Task Force. Yet in older adults, disability typically results from many small risks acting together with different people having a different pattern of multifactorial risk (U.S. Preventive Services Task Force, 2010). The importance of preventive hearing health care in primary care is discussed along with a screening strategy with targeted interventions designed to target older at risk adults.
\end{abstract}

\section{Introduction}

The aging of the world's population, with the number of older adults older projected to increase to $20 \%$ by 2050 will challenge countries, communities, and health care agencies to embrace preventive care as a means of promoting and supporting the joint goal of maintaining and improving the quality of life experienced as people age (Winkler, 2010). The demographic imperative as pertains to hearing is quite clear based on data accumulated over the years by the World Health Organization (Mathers, Smith \& Concha, 2003). Approximately 278 million people worldwide experience moderate to profound hearing

Correspondence: B.E. Weinstein, Ph.D., Professor and Executive Officer Au.D. Program Graduate Center, Cuny, 365 Fifth Ave, New York, USA.

E-mail: bweinstein@gc.cuny.edu

Key words: hearing impairment, screening.

(C) Copyright B.E. Weinstein, 2011

Licensee PAGEPress, Italy

Audiology Research 2011; 1:e12

doi:10.4081/audiores.2011.e12

This article is distributed under the terms of the Creative Commons Attribution Noncommercial License (by-nc 3.0) which permits any noncommercial use, distribution, and reproduction in any medium, provided the original author(s) and source are credited.

Parts of this work were presented at the "AHS 2010 - International Conference on Adult Hearing Screening”, Cernobbio (Italy), June 10-12, 2010. loss, making it the fifteenth most serious health problem in the world (Mathers, Smith \& Concha, 2003). Globally hearing loss is the second leading cause of years living with disability (YLD) behind depression and in fact it is a larger non-fatal burden than alcohol use, osteoarthritis and schizophrenia (Mathers, Smith \& Concha, 2003). The hearing loss population in America has reached 34 million with over 12 million adults 70 years of age and older who self report a hearing loss (Kochkin, 2009). Rarely occurring in isolation, hearing related problems increase the risk of functional decline especially in those with comorbid conditions. Tinnitus, which often accompanies hearing loss in older adults is a global problem as well, with significant psychological and functional consequences. Prevalence ranges from 4.5 to 19 percent globally with estimates varying with the definition and the characteristics of the sample (Nondahl, Cruickshanks, Wiley, Klein, Klein, Chappell, \& Tweed, 2010).

With regard to the demographic imperative, the hearing loss population has grown at the rate of $160 \%$ of the United States population growth, yet hearing health care is rarely included in the traditional medical exam, is not a screening priority, nor is it typically mentioned as a risk factor for cognitive decline or medical conditions such as falls. Patients tend to underreport symptoms of hearing impairment and as such it is one of eight problems which tends to be missed during the traditional medical exam. Further, there is gap between prevalence and treatment. Globally, less than $1 \%$ of hearing impaired individuals use hearing aids in developing countries, while in developed countries the rate ranges from 10 to $40 \%$ (Mathers, Smith \& Concha, 2003). Finally, hearing aid adoption rates have been static for more than 50 years despite advances in technology and more than 95 percent of those with hearing loss who could benefit from hearing aids do not use them (Kochkin, 2009).

The prevalence, co-morbidity and disabling effects of hearing loss coupled with the fact that hearing loss is underreported and underdiagnosed underscore the need for aggressive preventive programs which identify conditions such as hearing loss and related disorders which threaten traditional and non traditional health outcomes. In order to succeed, preventive programs must be collaborative and must target conditions which if identified can optimize care and reduce the burden of illness. Audiologists must partner with health care workers in primary care, notably, primary care physicians (PCPs) to promote preventive hearing health care. Collaborating with physicians may help remedy the fact that between $25-40 \%$ of hearing aid owners abandon use of hearing aids and that most adults wait about ten years before pursuing audiologic intervention. It is my conviction that across the health care continuum, adequate receptive ability is central to shared decision making, compliance with health instructions and accurate diagnoses, the latter being associated with positive outcomes and patient compliance. Not only do patient outcomes improve when barriers to communication are eliminated, but service delivery is made easier and more fulfilling with improved patient physician dialogue. In fact, Winkler (2010) p. 1355 in a recent issue of JAMA contends that outcomes should include improved functional and cognitive ability and mobility because those outcomes likely supersede traditional cardio- 
vascular outcomes in their importance to maintaining elders independence. Only through such adaptations to the new realities of the aging population will the goal of health care of not just bringing years to life but life to years be achieved.

Primary care physicians face a double edged dilemma. There is an increase in the number of chronically ill patients with co-morbid conditions. Yet most PCPS have not been trained to work in teams to provide care to those in need of their services. With so many infrastructure obstacles, the consensus among experts, is that high quality, cost-effective health care for older patients with multiple chronic conditions must be delivered as part of a comprehensive and multidisciplinary assessment. This should include evaluation of disabilities, cognitive abilities, health-related technologies, being used, health-related lifestyle habits, environmental risks, support systems, and other resources. The patient's relevant values and preferences for care, lifeplanning, and lifestyle, must inform many of the decisions being made (Boult \& Wieland, 2010; King \& Guralink, 2010). Pacala (2010) described an interesting approach to preventive health care namely matching preventive priorities with the patient's condition. For example, in healthy older adults with no evidence of disease or functional decline primary and secondary prevention should be the focus, whereas in individuals with chronic conditions or co-morbidities, the preventive priority should be tertiary prevention, accident prevention, and promotion of quality of care and patient engagement.. The definition of the three types of preventive care along with its relevance to hearing health care prevention is displayed in Table 1.

As is shown in Table 1, the health status of the target population should define the preventive activity. Hence healthy adults should be exposed to primary preventive activities, older adults with chronic conditions or co-morbid conditions are eligible for secondary or tertiary prevention and the frail elderly which comprise approximately $2-20 \%$ of the population of older adults may be eligible for tertiary prevention. In the case of the latter group, accident prevention, prevention of iatrogenic illness and quality of life are the important endpoints.

Prior to outlining the components of the proposed screening program, the target conditions for which I am proposing preventive activities, and the goals of the screening, I wish to elucidate the preconditions which should be met before mounting a screening program with some data to substantiate my position along with the theories which have informed my recommendations (Woolf, Jonas \& Kpalan-Liss, 2008; Yueh, Collins, Souza, Boyko, et al., (2010). Table 2 lists the preconditions by category including criteria the target condition, the intervention and the protocol should each meet before instituting a screening program for hearing related dysfunction (Woolf, Jonas \& Kaplan-Liss, 2008; Yueh, Collins, Souza, Boyko, et al., (2010). In my view, hearing health care meets most of the preconditions listed in Table 2, making it ripe for eligibility for routinized screening in primary care. Unfortaunately, data on preventive outcomes specific to

Table 1. Types of prevention (Gordon, 1983; Woolf, Jonas, \& Kaplan-Liss, 2008).

\section{Type of prevention Definition}

\begin{tabular}{lll} 
Primary & $\begin{array}{l}\text { Prevent or inhibit illness or disability from occurring } \\
\text { in the first place- prior to biologic onset of the disease }\end{array}$ & $\begin{array}{l}\text { Practice healthy hearing behaviors such as noise protection to } \\
\text { protect against impairment and disability }\end{array}$ \\
$\begin{array}{l}\text { Decondect impairment or disease in an early stage in an effort } \\
\text { to minimize consequences and complications; detect condition } \\
\text { before symptomatic; practiced after the condition is recognized } \\
\text { but before if has caused disability or suffering }\end{array}$ & $\begin{array}{l}\text { Screening programs for early detection before } \\
\text { condition is noticeable and before } \\
\text { complications begin to present }\end{array}$ \\
\hline Prevent progression of disease and attendant suffering; & $\begin{array}{l}\text { Promote management in people with co-morbidities; } \\
\text { practiced after suffering and disability are }\end{array}$ & $\begin{array}{l}\text { attempt to reduce disability and restore function and } \\
\text { self-sufficiency in the chronically ill; aim to improve the quality } \\
\text { of life for people with various diseases by limiting complications } \\
\text { and disabilities, reducing the severity and progression } \\
\text { of disease, and providing rehabilitation (therapy to restore } \\
\text { functionality and self-sufficiency) }\end{array}$ \\
\end{tabular}

Table 2. Preconditions to be met before initiative a screening program (Woolf, Jonas \& Kpalan-Liss, 2008; Markle-Reid, Keller \& Browne, 2010; Frankish, Lovato, \& Poureslami, 2007; Pacala 2010).

\section{Preconditions}

The target condition

The condition must be important based on prevalence and incidence rates in the segment of the population targeted

The burden of illness must be substantial in that the consequences of the condition must be serious for the individual, family members, and/or society

The natural history of the target condition must be such that adequate time is available for successful interventions to be instituted and to succeed

Available Interventions

Available interventions should be effective and tailored such that they are proven to improve function, quality of life, and quality of care.

Available interventions should be accessible, beneficial, acceptable, culturally relevant, and the community must have the capacity to absorb referrals The likelihood that the target population will comply with and benefit from the outcome of the intervention recommended should be high

The screening protocol

Must be acceptable to the health care provider performing the screen

Should be sustainable with effectiveness demonstrated through randomized trials

Must include referral to community agencies and health care providers with the necessary manpower to provide follow up services to insure that identified needs are met

Should include culturally appropriate evidence based compliance improving strategies

Should be inexpensive, brief, easy to administer, reliable, acceptable, sensitive, specific, ethical so that there is no risk of physical harm to the patient, appropriate for the segment of the population being targeted

Include a systems based approach involving information technology, evidence based guidelines, community involvement and incentives for preventive care 
older adults in general and to hearing health care in particular are rare, as are data on the cost benefit of preventive hearing health care, hence the Preventive Services Task Force does not support hearing health care screening (U.S.P.S.T.F., 2010). Evidence which would help provide data in support of hearing health care screening would be a determination that total potential health benefits from hearing care is higher among those who received the service than from among those who have not received the service (Maciosek, Coffield, Edwards, Flottemesch, Goodman \& Solberg, 2006). The health behavior theories listed in Table 3 informed my selection of the components of the protocol. The theories are divided among the Social Learning Theories and Cognitive Theoretical Models. The Health Belief Model posits that perception of susceptibility to and seriousness of the condition will influence willingness to comply (Becker, 1974). Milstein \& Weinstein (2007) used this model as the basis for the screening protocol they studied. The Transtheoretical Stages of Change Model also informed their work in the area of screening (Prochaska \& DiClemente, 1982). According to this model, readiness to comply with behavior change (e.g. participate in a screening program and comply with recommendations) depends on the psychologic stage the individual finds $\mathrm{him} /$ herself in ranging from the precontemplation, to the action stage and culminating in the maintenance or relapse phase. This theory informed my conviction of the importance of tailored interventions as a means of influencing rates of compliance and movement toward the most effective intervention. The Social Learning Theories are of relevance when discussing the intervention/rehabilitation options following screening which can range from behavioral counseling for the patient and caregiver, educational counseling for patient and caregiver regarding amplification strategies, hearing assistive technologies, communication strategies or participation in communication program. The epidemiologic literature is replete with evidence of the integral role these theories play in the design of effective health promotion programs. Finally, my proposal for a comprehensive screening program was based in part on an informal systematic review of the literature on screening programs targeting older adults including the most recent work of Yueh, et al., (2010), the most comprehensive trial to date. Many of the studies have employed a self report questionnaire, a pure-tone impairment screen, and referrals to an audiologist for consideration of hearing aids. In the case of the former, the screening version of the Hearing Handicap Inventory for the Eldelry (HHIE-S) was used by most investigators, the Audioscope ${ }^{\mathrm{TM}}$ or an audiometer were used for the impairment screen, and the interventions recommended were limited mostly to and audiologic evaluation for consideration of hearing aids rather than interventions targeted to the communicative and lifestyle needs of the person being screened. Interestingly, in most studies compliance with the recommendation for a follow-up was low and the majority of those recommended hearing aids did not obtain them.

The four arm randomized trial conducted by Yueh et al., (2010) had a as a goal the determination of which screening strategy led to the most patients wearing hearing aids. Interestingly, in their sample only $18 \%$ of participants (outpatient veterans) screened positive for hearing impairment with the Audioscope, 59 percent screened positive for hearing loss based on HHIE-S scores, and 63 percent screening positive for hearing loss with the dual arm screen. Further, a slightly higher percentage of those who failed the Audioscope ${ }^{\mathrm{TM}}$ screen were fit with hearing aids than those who failed the HHIE-S screen, yet only 4 percent of those screened with the HHIE, 6 percent of those screened with the Audioscope, and 7 percent of those in the dual arm wore hearing aids after one year. As only 3 percent of those in the control group who were not screened wore hearing aids after one year, the value of the screening in terms of increasing hearing aid use might be called into question. While the authors, concluded that the pure-tone screen using the Audioscope was sensitive to identifying those with hearing loss, the HHIE and combined approach had several advantages, as well. Individuals were seen by the audiologist in a pre-symptomatic phase before the impairment presented itself, a combined approach to screening led to more early visits to the audiologist and to a higher percentage of hearing aid fittings and many participants indicated that had they not undergone the screen, they would not have sought out an evaluation or opted for treatment (Yueh, et al., 2010).

\section{The screening program}

In keeping with the aforementioned guiding principles, the first step in designing a strategy is to define the goal of the comprehensive screening program. The goals of the proposed program are in keeping with the thinking of Yueh and his colleagues (2010) and the philosophy of the U.S. Preventive Services Task Force (2010). First, we want to target patients who are willing to seek and adhere to treatments that will improve health outcomes and optimize patient function. Second, we want to target individuals with co-comorbid conditions in whom identifying a hearing related conditions can help physicians better manage the patient, improve the patient's ability to understand health instructions, and participate in shared decision making (U.S.P.S.T.F., 2010) The next decision to be made in the design of a program relates to the health professional who will conduct or coordinate the screening program. In my view the primary care physician (PCP) should be the responsible party with the audiologist educating the professional regarding protocols and strategies. The $\mathrm{PCP}$ is the gatekeeper for entry into the healthcare system in that $80 \%$ of older adults make at least one annual physician visit and older adults with multiple chronic conditions make multiple visits. Further, PCPs are the most credible source for health matters in that patients who are successful in changing their health behavior credit advice from their physician as the motivational force (Glasgow \& Goldstein 2008). The global data on annual physician visits for preventive care, based on a recent report by the Organization for Economic Cooperation and Development (2010), are of interest. While in Japan the average number of annual consultations for preventive care is 13.6, in Germany it is 7.3 and in the United States it is 3.8. So, the concept of the physician as gatekeeper is a global phenomenon.

Table 3. Health behavior theories underlying successful health promotion (Glasgow \& Goldstein, 2008).

\section{Social leaming theories}

Behavioral capacity - participant must have the skills necessary for performing the desired behavior required to complete the screening and the recommended intervention

Efficacy expectations - participant must have confidence in their ability to successfully carry out the recommended course of action

Outcome expectations - participant must believe that adherence to recommended interventions will have desired effects, consequences, or actions;

\section{Coginitive theoretical model}

Health Belief Model - the inner world or motivational level of the participant influences their health seeking behavior and typically here must be a perceived incentive to take action (Becker, 1974)

Transtheoretical stages of change model - individuals progress through a sequence of discrete stages before embracing new behaviors (Prochaska \& DiClemente, 1982)

The expectation is that the action will contribute to further health and well being and this information must be conveyed via materials disseminated as part of the screen or via counseling at the time of the screen. 
Finally for physicians to carry out their health examination effectively and to involve the patient in shared decision making adequate receptive abilities is key. Table 4 includes a list of five aspects of the work of physician's which demands adequate receptive communication and underscores why the PCP should be incentivized to collaborate.

It is clear that as people age and live longer, physicians are spending more time caring for older adults with multifactorial conditions including depression, social isolation, cognitive impairments, vision loss, cardiovascular disease and managing hearing loss in this population can influence morbidity and mortality. Since cognitive impairment (CI) is an independent risk factor for falls and $\mathrm{CI}$ is associated with hearing loss and auditory processing, screening activities targeting those at risk are important. In addition, hearing loss is a factor which co-occurs in most disease which compromise morbidity and mortality including diabetes, vascular disease, depression, and heart failure. Older adults with hearing loss, vision impairment, and dual sensory impairment have higher rates of mortality, and ADL dependency than those without sensory loss. Crews and Campbell (2004) examined the 1994 Second Supplement on Aging data and uncovered that older adults with hearing loss have higher rates of co-morbid and secondary conditions than those without and the likelihood of co-morbid and secondary conditions in people with vision and hearing loss is greater in those without dual sensory loss. This is shown clearly in Table 5 . In addition to the data in Table 5, Crews and Campbell (2004) noted that people with hearing loss and vision impairment experienced substantial difficulty sustaining social participation activities, with fewer interactions with friends and relatives. Finally, individuals with hearing loss had a higher likelihood of being depressed and confused than those without hearing loss. Since the presence of hearing loss can alter the course and treatment of an individual with multifactorial conditions, it behooves PCPs, to early on identify and refer those with hearing impairment as this could alter the quality of care and the nature of health outcomes.

Table 6 includes the features of the holistic screening protocol I am advocating. A unique feature is that integral to its success is a patientclinician partnership. The unique features include a mulit-component protocol, a physician tool box and targeted interventions. The first step is a global and hearing related assessment. The high risk profile grows out of the high prevalence of co-morbidities in older adults. It is designed to identify potential risk factors for hearing impairment. The form I developed for the assessment is shown in Table 7. It includes medical conditions associated with hearing impairment as identifying at risk individuals individuals has the potential to improve quality of

Table 4. Non-traditional aspects of a primary care which rely on good functional communication (Leipzig, Granville, Simpson, Brownell, Sauvigne, \& Soriano, 2009).

Communicate key components of a safe discharge plan including accurate discussion of medications and plans for follow-up

Conduct regular assessments into home safety and medication use to help prevent falls

Conduct cognitive and depression screens as part of routine practice or in advance of surgical procedures

Conduct a cognitive assessment should their be suspicion of memory impairment or Alzheimer's Disease

Discuss end of life care, advanced directives, possible palliative care

Table 5. Likelihood of comorbid condition in those with hearing loss and dual sensory impairment (Crews \& Campbell, 2004).

Condition Likelihood of presenting with co-morbid condition among those with hearing loss as compared to those without hearing loss

Experienced falls $\quad 1.7$ times more likely to have experienced falls in past 12 months $\quad$ (statistically significant)

Heart disease $\quad 1.7$ times more likely to report heart disease (significant) Hypertension Significantly higher rates of hypertension

Broken Hip Significantly higher rates of broken hips
Likelihood of presenting with co-morbid condition among those with vision and hearing loss as compared to those without dual sensory loss

3 times more likely to have fallen in past 12 months (significant)

2.4 times more likely to report heart disease (significant) 1.5 times more likely to report hypertension (significant) 2 times more likely to have broken a hip (significant)

Table 6. Components of screening protocol (Whitlock, Orleans, Pender \& Allan, 2002).

\begin{tabular}{|c|c|c|}
\hline Physician tool box & Multicomponent protocol & $\begin{array}{l}\text { Outcome based targeted intervention } \\
\text { Recommendations - Options }\end{array}$ \\
\hline Screening protocol & Global risk profile assessment & Healthcare provider advice - promote hearing health literacy \\
\hline Referral guidelines & Performance based screen for risk for hearing impairment & $\begin{array}{l}\text { Complete audiologic } \\
\text { Evaluation } \\
\text { Provide information regarding community } \\
\text { resources for hearing and fearing aid evaluaion and fittings }\end{array}$ \\
\hline $\begin{array}{l}\text { Multifaceted and tailored } \\
\text { interventions }\end{array}$ & Screening test of functional communication impairment & $\begin{array}{l}\text { Behavioral counseling re communication strategies and } \\
\text { environmental aids for small groups, television and music, } \\
\text { and warning sounds; counseling re home hazards } \\
\text { modifications; caregiver counseling regarding communication } \\
\text { strategies and technologies }\end{array}$ \\
\hline \multicolumn{2}{|c|}{$\begin{array}{l}\text { Physician education-conducted } \\
\text { by the audiologist }\end{array}$} & $\begin{array}{l}\text { Multifactorial screen } \\
\text { Risk evaluation } \\
\text { Behavioral counseling } \\
\text { Interventions and outcomes }\end{array}$ \\
\hline \multicolumn{2}{|c|}{ Follow-up guidelines (e.g. telephone or email contacts) } & $\begin{array}{l}\text { Written recommendations } \\
\text { Follow-up } \\
\text { Five A's framework: construct-assess, advise, agree, assist, } \\
\text { and arrange should be the basis for written recommendations }\end{array}$ \\
\hline
\end{tabular}


patient centered care and to reduce the burden of illness. For example, hearing impairment is significantly associated with depression and the relationship is independent of age (Yueh, Shapiro, MacLean Shekelle, 2003). Further, hearing loss is common in individuals with diabetes and diabetes is an independent risk factor for hearing impairment (Bainbridge, Hoffman \& Cowie, 2008). The risk profile assessment can be included in the intake patients may complete in the waiting room or in advance of reporting to the physician's office. Responses to the profile can trigger the need for a routine screen and the outcome of the screen together with information from the risk assessment can inform the patient provider discussion regarding follow-up options. Similarly, responses may alter the course of the medical examination or how the physician communicates with the patient during the examination. Perhaps, a personal amplifier might be used to insure that the assessment is not contaminated by hearing loss or to facilitate the flow of the medical exam and recommendations regarding medical regiments. In contrast, if the patient is free of chronic conditions, appears to be a relatively healthy older adults, and fails the physiologic and self report
Table 7. Hearing health risk appraisal form.

\section{Health condition}

1. Do you smoke cigarettes

2. Do you or a family member believe that you have 10 difficulty hearing and understanding others?

3. Have you ever been told that you now have diabetes mellitus?

4. Have you been told that you have cardiovascular sease at this time?

5. Have you been told that you now have arthritis?

6. Are you taking aminoglycoside antibiotics, cisplatin, anti inflammatory agent or loop diurectics?

7. Have you had a fall within the past year? 10

8. Have you been told that you have low vision or blindness?

9. Have you been told that you are suffering from depression?

Table 8. Screening for functional communication impairment.

Instructions: The purpose of this questionnaire is to identify any problems you are having which may relate to your ears - including hearing difficulties or tinnitus (ringing, buzzing, noises in your ears/head). Please circle either Yes, Sometimes, or No for each question. If you use hearing aids, please answer the way you hear without using the hearing aids. If you are not experiencing any hearing difficulties, please mark No for each item.

\section{Patient ID:}

\begin{tabular}{|c|c|c|c|c|c|}
\hline \multicolumn{2}{|r|}{ Scoring Values for Each Item } & 4 & 2 & 0 & NA \\
\hline $\mathrm{H} \cdot 1$ & $\begin{array}{l}\text { Does a hearing problem cause you difficulty when listening } \\
\text { to the television or to the radio }\end{array}$ & Yes & Sometimes & No & \\
\hline MI-1 & $\begin{array}{l}\text { Is it important for your hearing problem to cause you less } \\
\text { difficulty when listening to the television or radio? }\end{array}$ & Yes & Sometimes & No & \\
\hline $\mathrm{H} \cdot 2$ & $\begin{array}{l}\text { Does a hearing problem cause you difficulty when visiting } \\
\text { friends, relatives or neighbors? }\end{array}$ & Yes & Sometimes & No & \\
\hline H.3 & $\begin{array}{l}\text { Does a hearing problem cause you to feel frustrated when } \\
\text { talking to members of your family? }\end{array}$ & Yes & Sometimes & No & \\
\hline MI-2 & $\begin{array}{l}\text { Is it important for you to feel less frustrated by a hearing } \\
\text { problem when talking to members of your family? }\end{array}$ & Yes & Sometimes & No & \\
\hline MI-3 & $\begin{array}{l}\text { Is it important for a hearing problem to cause you less } \\
\text { difficulty when visiting with friends, relatives, or neighbors? }\end{array}$ & Yes & Sometimes & No & \\
\hline \multicolumn{6}{|c|}{ 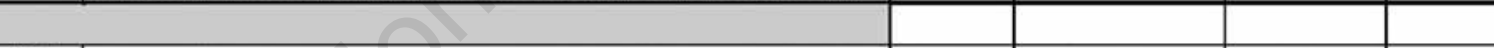 } \\
\hline T.1 & Do you feel that you can no longer cope with your tinnitus? & Yes & Sometimes & No & \\
\hline T-2 & $\begin{array}{l}\text { Because of tinnitus, do you have trouble falling asleep or } \\
\text { staying asleep at night? }\end{array}$ & Yes & Sometimes & No & \\
\hline T-3 & Do you feel that you cannot escape your tinnitus? & Yes & Sometimes & No & \\
\hline \multicolumn{2}{|r|}{$\begin{array}{l}\text { How confident do you feel that you are ready to take the } \\
\text { necessary steps to help you hear/understand better in } \\
\text { difficult listening situations (Please circle number that } \\
\text { applies)? }\end{array}$} & $\begin{array}{l}\text { Not } \\
0 \quad 1\end{array}$ & 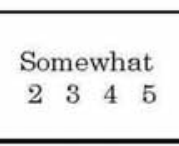 & $\begin{array}{l}\text { Very } \\
67\end{array}$ & \\
\hline \multicolumn{6}{|c|}{ TOTAL SCORE } \\
\hline & $\begin{array}{c}\text { In the box below, please circle the number tha } \\
\text { The severity of the probler } \\
\text { Not at all severe }\end{array}$ & corres & onds with: & & \\
\hline Hea & ring Loss: & 6 & 7 & & \\
\hline Tin & nitus: & 6 & 7 & & \\
\hline
\end{tabular}


screen, this might lead to a discussion of intervention options to consider and referral for a complete hearing test. The hearing health risk appraisal form should be easy to score and available in multiple languages depending on caseload considerations. As health risks change over time, it would be important for these to be completed annually. The presence of any of the conditions should trigger consideration of a hearing screen. Gates \& Mills (2005) recommended that screening for hearing loss be completed as part of the annual physical examination. In their scientific review of screening for hearing loss in primary care, Yueh, Shapiro, MacLean, \& Shekelle (2003), contend that many cases of hearing loss are treatable in the primary care setting, and "recognition of hearing loss facilitates referral to appropriate hearing professionals for treatment that may lead to better quality of life, p. 1984). Finally, in the summary of the updated American and British Geriatrics Societies (2010) they recommend that physicians conduct a multifactorial risk assessment of community dwelling older persons who report recurrent, difficulty with gait or balance, or seek medical attention because of a fall. As available data suggest that hearing loss certainly qualifies as a risk factor, given correlations between pure tone levels, dizziness and sense of balance, screening this population for hearing impairment is justified. Since most PCPs tend to only refer when patients complain of having hearing and balance difficulties, and most are not familiar with reliable and valid tools used in screening for hearing related disorders, educational and informational outreach campaigns targeting physicians regarding screening, the consequences of hearing loss, and of course providing them with tools to screen is critical if screening programs are to be effective (Johnson, Danhauer, Koch, Celan, Lopez \& Williams, 2008).

The next step is the screen for risk for hearing loss and functional communication impairments. A twofold screen is important as the goal is to identify those with co-morbidities who may have a hearing impairment as a correlate and to pinpoint the extent of the functional communication deficits so as to match behavioral characteristics including stages of readiness and motivation level the appropriate follow-up recommendations. Tailoring the recommended intervention is key in terms of success and is in keeping with current thinking in the area of epidemiology. In keeping with the work of Ventry \& Weinstein (1983) and as corroborated by many other investigators (Yueh, et al.,2003), I recommend an impairment screen at 1000 and $2000 \mathrm{~Hz}$ at $40 \mathrm{dHL}$ using the Audioscope ${ }^{\mathrm{TM}}$. Regarding the self-report screener, a modification of the HHIE-S, known as the SCFI (Screening for Functional Communication Impairments) is shown in Table 8. It is based on discussions with Therese Hnath-Chisolm and Joe Montano and an extensive review of the literature on screening and the important role of instrinsic motivation and self efficacy as a determinant of compliance. The data from the self report can inform behavioral change counseling integrated into the primary care setting as part of the screening. Note that I have included tinnitus, as it is a prevalent and disabling hearing related condition. Embedded in the questionnaire are items that target health behaviors which predispose an individual to successful behavior change. These unique items are defined in Table 9 (Whitlock, Orleans, Pender, Allan, 2002). Based on preliminary data, if the sum of the $\mathrm{H}+\mathrm{MI}+\mathrm{SE} \geq$ to 8 the patient should be referred for a complete audiological evaluation. If $\mathrm{T} J=4$ the patient should be referred for an Otolaryngologic work-up. A pass on the SFCI and a fail on the pure-tone should include dissemination of an information packet which includes educational material about intervention options and their value, tips on communicating, tips for home safety, information about hearing assistive technologies, along with a referral for a baseline audiologic evaluation, and caregiver counseling regarding communication and hearing assistive technologies. Finally, in the case of a fail on the Audioscope ${ }^{\mathrm{TM}}$ screen, the PCP may consider using a personal amplifier to facilitate effective communication and to insure that the patient is able to understand health instructions and can participate in shared decision mak-
Table 9. Key correlates of successful behavioral change included in SFCI (Whitlock, Orleans, Pender, \& Allan 2002).

Strongly wants and intends to change - Motivational Interviewing Has requisite skills and self-confidence to make a change - Self efficacy (SE)

Feels positively about the change and believes it will result in meaningful benefit - SE

Acknowledges existence of a hearing related condition (Tinnitus or hearing)

ing regarding goals of care or end of life decisions (U.S.P.S.T.F., 2010). The latter would be provided by the audiologist and included as one component of the Physician's Tool Box. As noted in Table 6, behavioral counseling, written recommendations regarding patient disposition, and telephone or email follow-up regarding compliance are key components of the screening protocol if outcomes are to be positive as is monitoring patient response to treatment. Similar to the tool kit developed for PCPs for screening for depression, the tool box should include: i) description of instruments for recognizing and diagnosing hearing impairment; ii) materials for educating patients about hearing impairment, tools for assessing treatment preferences, and suggestions for engaging in the process of caring for their hearing; iii) evidence-based guidelines iv) management tools for communicating with the hearing impaired and explications regarding available technologies, strategies and community resources and v) guidelines for the caregiver (Macarthur Initiative, 2011).

\section{Conclusions}

In sum, hearing screening of older adults is important from the perspective of improving traditional health outcomes such as quality of care and quality of life and less traditional outcomes such as effect of hearing impairment on the caregiver. PCPs are key partners if audiologists are to reach those with undiagnosed and unremediated hearing impairments. As with neonatal screening, the role of the audiologist in this enterprise is critical and integral to the efficacy of screening initiatives. Audiologists must educate PCPs about screening protocols, procedures, and competencies along with discussing the prevalence and consequences of unidentified hearing los to insure that they appreciate the rationale underlining preventive hearing health care. Borrowing from the 5As of counseling, physicians must come to understand what I call the six A's of hearing health care promotion: i) advice regarding rationale for and protocols screening, ii) assessment of readiness to take action, iii) agree to targeted counseling approaches regarding follow-up, iv) arrange for community resources to accommodate referrals, v) ask the patient about their hearing status from the outset and vi) assist the patient with unraveling their susceptibility to hearing loss and the consequences, if untreated (Glasgow, Emont \& Miller, 2006). The sustainability of any efforts at reducing the gap between prevalence, diagnosis, and treatment of hearing related dysfunction will rest in large part on the knowledge base of patient and caregiver so this component of any screening strategy might be considered as primary and secondary prevention measures and an important component of the screening activities conducted by PCPS. Regarding, physician competencies, hearing status is key to seven domain competencies considered integral to the practice of medicine with older adults. These content areas include: medication management; falls, balance and gait disorders; hospital care for elders; cognitive and behavioral disorders; atypical presentation of disease; health care planning and promotion; and palliative care (Leipzig, Granville, Simpson, 
Brownell, Sauvigne, \& Soriano, 2009). Perhaps, working with colleagues from a variety of disciplines, universal screening for hearing related disorders will become a reality thanks to the vision of the conveners of the AHS-2010 conference.

\section{References}

Bainbridge, K., Hoffman, H., \& Cowie, C. 2008. Diabetes and hearing impairment in the United States: Audiometric evidence from the National Health and Nutrition Examination Survey, 1999 to 2004. Annals of Internal Medicine, 149, 1-10.

Becker, M. 1974. The Health Belief Model and Personal Health Behavior. Thorofare, NJ: Charles B. Slack, Inc.

Boult, C., \& Wieland, G. 2010. Comprehensive Primary Care for Older Patients With Multiple Chronic Conditions: : "Nobody Rushes You Through. " JAMA. 304: 1936-1943.

Carol, R. 2005. How to conduct a "Welcome toe Medicare" visit. Family Practiice Management. 12, 27-29, 31-32.

Crews, J. \& Campbell, V. 2004. Vision impairment and hearing loss among community dwelling older Americans: Implications for health and functioning. American Journal of Public Health. 94:823829.

Frankish J, Lovato C, Poureslami, I. 2007. Models, Theories and Principles of Health Promotion in Multicultural Populations. In: R. Huff \& M. Kline, Editors, Promoting Health in Multicultural Populations, Second Edition. New York: Sage.

Gates, G., \& Mills, J. 2005. Presbycusis. Lancet, 366: 1111-1120.

Glasgow,R., Emont, S. \& Miller, D. 2006. Assessing the delivery of the five 'As' for patient-centered counseling. Health Promotion International. 21: 245-255.

Glasgow, R. \& Goldstein, M. 2008. Introduction to the Principles of Health Behavior Change. In: S. Woolf, S. Jonas, \& E. Kaplan-Liss (Eds.). Health promotion and disease prevention in clinical practice. Second Edition. Maryland: Lippincott, Williams \& Wilkins.

Gordon, R. 1983. An operational classification of disease prevention. Public Health Report. 98:107-109.http:/www.ncbi.nlm.nih.gov/ pmc/articles/ PMC1424415/?page=1Accessed January 18, 2011.

King, A. \& Giuralnik, J. 2010. Maximizing the Potential of an Aging Population. JAMA. 304: I1944-I1945.

Leipzig, R., Granville, L., Simpson, D., Brownell, Sauvigne, A., \& Soriano, R. 2009. Keeping Granny Safe on July 1: A Consensus on Minimum Geriatrics Competencies for Graduating Medical Students. Academic Medicine. 84:604-610.

Johnson, C., Danhauer, J., Koch, L., Celan, K., Lopez, Il, Williams, V. 2008. Hearing and balance screenig and referrals for Medicare patients: A national survey of primary care physicians. Journal American Academy of Audiology. 19, 171-190.

Kochkin, S. 2009. Marke-Trak VIII: 25 year trends in the hearing health market. Hearing Review. 10: 12-31.

Macarthur Initiative on Depression \& Primary Care 2011. http://www.depression-primarycare.org/clinicians/toolkits/ Accessed January 18, 2011.

Maciosek, M., Coffield, A., Edwards, N., Flottemesch, T., Goodman, M., \& Solberg, L. 2006. Priorities among effective clinical preventive services: results of a systematic review and analysis. American Journal of Preventive Medicine. 1:52-61.

Markle-Reid, M., Keller H.H., Browne, G. 2010. Health promotion in the community living older adult. H. Fillit, K. Rockwood, K. Woodhouse (eds.) Brocklehurst Textbook of Geriatric Medicine and Gerontology, 7th Ed.

Mathers, C., Smith, A., \& Concha, M. 2003. Global burden of hearing loss in the year 2000 (online). Geneva: World Health Organization. Available at: http://www.who.int/healthinfo/statistics/bod_hearingloss.pdf. [Accessed 16 January 2011].

Milstein, D. \& Weinstein, B. 2007. Milstein, D. and Weinstein, B. Universal Hearing Screening in Adults: If Not Now, When? Communicative Disorders Review. 1: 107-119.

Nondahl, D., Cruickshanks, K., Wiley, T., Klein, R., Klein, B. \& Tweed, T. 2002. Prevalence and 5-year incidence of tinnitus among older adults: the epidemiology of hearing loss study. Journal of American Academy of Audiology, 13, 232-331.

Maciosek, M., Coffield, A., Edwards, E. , Flottemesch, T., Goodman, M., \& Solberg, L. 2006. Priorities among effective clinical preventive services: results of a systematic review and analysis. American Journal of Preventive Medicine. 31: 52-61.

Nondahl, D., Cruickshanks, K., Wiley, T., Klein, B., Klein, R. , Chappell, R., Tweed, T. 2010. The ten-year incidence of tinnitus among older adults. International Journal of Audiology. 49, 580-585.

Organization for Economic Cooperation and Development 2010 http://www.oecd.org/document/44/0,3746,en_2649_37407_2085228 1_1_1_37407,00.html

OECD Health Data 2010: Statistics and Indicators. Accessed January $18,2011$.

Ouslander, J. 2010. A murmur of music. JAMA. 304: 1875

Pacala, J. 2010. Preventive and anticipatory care. In: In: H.Fillit, K. Rockwood, \& K. Woodhouse (Eds.). Brockelhurst's Textbook of Geriatric Medicine and Gerontology. Seventh Edition. Pennsylvania: Saunders Elsevier.

Prochaska, J. \& DiClemente, C. 1982. Thranstheoretical therapy-toward a more integrative model of change. Psychotherapy: Theory, Research and Practice. 19: 276-288.

Reuben, D., Mui, S., Moore, D., Moore, A. \& Greendale, G. 1999. The prognostic value of sensory impairment in older adults. Journal American Geriatrics Society. 47: 930-935.

Solodar, H. \& Chappell. 2005. "Welcome to medicare" preventative exam includes hearing and balance screening Audiology Today 17, 49.

Ventry, I. \& Weinstein, B. 1983. Identification of elderly people with hearing problems. ASHA. 25: 37-42.

U.S. Preventive Services Task Force. U.S.P.S.T.F., 2010. www.uspreventiveservicestaskforce.org. Reconsidering the approach to prevention recommendations for older adults. Accessed January 18, 2011.

Whitlock, E. Orleans, C., Pender, N. \& Allan, J. 2002. Evaluating primary care behavioral counseling interventions: an evidence-based approach. Am J Prev Med. 22:320-3.

Winkler, M. 2010. Preserving Function, Improving Care. JAMA. 304: 1954-1955.

Woolf, S., Jonas, S. \& Kaplan-Liss, E. 2008. Health Promotion and Disease Prevention in Clinical Practice - Second Edition. Philadelphia: Wolters Kluwer/ Lippincott Williams \& Wilkins.

Summary of the Updated American Geriatrics Society/British Geriatrics Society Clinical Practice Guideline for Prevention of Falls in Older Persons. 2011. J Am Geriatr Soc 59:148-157.

Yueh, B. Collins, M., Souza, P., Boyko, A., Loovis, C., Heagerty, P., Liu, C., \& Hedrick, S. 2010. Long-Term Effectiveness of Screening for Hearing Loss: The Screening for Auditory Impairment -Which Hearing Assessment Test (SAI-WHAT) Randomized Trial. Journal of the American Geriatrics Society. 58: 427-434.

Yueh, B., Shapiro, N. MacLean, C., Shekelle, P. 2003. Screening and management of adult hearing loss in Primary Care. JAMA. 289: 1976-1985. 